\title{
Penetration Capacity Calculation for Wind Farms Considering Random Output
}

\author{
Haitao Liu ${ }^{1, a}$, Jian Su ${ }^{1, b}$, Pipei Zhang ${ }^{2, c}$, Hao Bai ${ }^{2, d}$, Shihong Miao ${ }^{2, e}$ \\ 1 China Electric Power Research Institute, Beijing100192, China; \\ 2 State Key Laboratory of Advanced Electromagnetic Engineering and Technology, Huazhong \\ University of Science and Technology, Wuhan430074, China. \\ a Ihtcn@epri.sgcc.com.cn; \\ b sujian@epri.sgcc.com.cn; '15549438042@163.com; 'baihao@hust.edu.cn; 'shmiao@hust.edu.c
}

$\mathrm{n}$

Keywords: wind farm, random output, probabilistic load flow, penetration capacity

\begin{abstract}
The majority of the distribution network is supplied by unilateral power and has a radial distribution, wind farms connected into grid will inevitably bring a certain extent on static voltage of the distributed grid. Furthermore, the randomness of wind farm output will cause the node voltage fluctuations. This paper firstly studies the random flow calculation method including wind power, and analyzes the probability distribution of the grid voltage when wind farms with different capacity accessing by combining the Newton-Raphson method with probability theory. Then establishes a computing model of penetration capacity of wind farms with the constrain of the static voltage stability range. For different values of confidence level $\alpha$, the results of penetration capacity are different, then we can choose the value $\alpha$ flexibility according to the distribution network security requirements.
\end{abstract}

\section{I . Introduction}

The access of the wind farms influences the size and direction of power flow, which makes the static voltage of distribution network change. Active power that wind farms transport to the access point, reduces the active power that the infinite system delivers to loads in grid, thereby reducing the voltage drop, making the overall voltage level to enhance in distribution network. While with the wind farm output increasing, a large amounts of reactive power will be consumed, leading to a voltage drop near the access point, and even destroy static voltage stability [1]-[3].Distribution Network static voltage stability depends to some extent on the access point and capacity size of wind power. The closer wind farm to the end node and the greater penetration capacity, the more significant impact on distribution voltage [4]-[6].

The above conclusions are based on wind power with a constant output, but in fact, the output of wind farms is closely linked to the wind speed and direction within a certain time. Therefore, when a certain capacity of wind power connected to grid, its active efforts will not always maintain the rating at all time, but change with the random fluctuation of energy, making the voltage of each node in random fluctuations. To be a comprehensive analysis on the system static voltage level changes brought by wind power, and its penetration capacity calculation, we must consider the impact of wind power output randomness.

\section{II . Random Flow Calculation Method including Wind Power}

Semi-invariant and Gram-Charlier series. Set the random variable $x$ with the probability density function $\mathrm{f}(\mathrm{x})$,and its $\mathrm{v}$ order moment can be calculated by the following equation:

$$
\alpha_{v}=\int_{-\infty}^{+\infty} x^{v} f(x) d x
$$

Semi-invariant is an attribute of random variable itself, that can be calculated from the 
corresponding order moments. For random variable with unknown probability density, its semi-invariant can be obtained from the homogeneous and additivity [7].

Gram-Charlier series may obtain the probability density function of an unknown random variable using each order semi-invariant. Gram-Charlier expansions are shown as follows:

$$
\begin{aligned}
& F(x)=\Phi(x)+c_{1} \Phi^{\prime}(x)+c_{2} \Phi^{\prime \prime}(x)+c_{3} \Phi^{\prime \prime \prime}(x)+\cdots \\
& f(x)=\phi(x)+c_{1} \phi^{\prime}(x)+c_{2} \phi^{\prime \prime}(x)+c_{3} \phi^{\prime \prime \prime}(x)+\cdots
\end{aligned}
$$

Where, $\phi(x)$ and $\Phi(x)$ are respectively the probability density function and distribution function of standard normal distribution. The coefficients $\mathrm{cn}(\mathrm{n}=1,2,3, \ldots)$ can be obtained by each order of semi-invariant [8].

Weibull distribution and wind farm output model. Weibull distribution is often used to fit the wind speed distribution in a certain region [9], its probability density expression is:

$$
f(v)=\frac{k}{c} \cdot\left(\frac{v}{c}\right)^{k-1} \cdot \exp \left[-\left(\frac{v}{c}\right)^{k}\right]
$$

Where, $\mathrm{v}$ is the wind speed, $\mathrm{c}$ and $\mathrm{k}$ is the scale parameter and shape parameter.

Expressions between wind farm active output and wind speed is shown below:

$$
P_{W}=\left\{\begin{array}{l}
0, v \leq v_{c i} \\
k_{1} v+k_{2}, v_{c i} \leq v \leq v_{r} \\
P_{r}, v_{r} \leq v \leq v_{c o} \\
0, v_{c o} \leq v
\end{array}\right.
$$

Where, $\mathrm{P}_{\mathrm{r}}$ is the rated output of the wind turbine, $\mathrm{v}_{\mathrm{ci}}$ called cut-in speed, $\mathrm{v}_{\mathrm{r}}$ called the rated wind speed, $v_{\text {co }}$ called the cut-out wind speed. According to equation (3) and (4) we can obtain the probability density function of wind farm active output [10].

$$
f\left(P_{W}\right)=\frac{k}{k_{1} C} \cdot\left(\frac{P_{W}-k_{2}}{k_{1} C}\right)^{k-1} \cdot \exp \left[-\left(\frac{P_{W}-k_{2}}{k_{1} C}\right)^{k}\right]
$$

Linear model of flow equations. The node power equation and branch flow equation of distributed network are summarized as follows:

$$
\begin{aligned}
& S=f(x) \\
& Z=g(x)
\end{aligned}
$$

Where, $\mathrm{S}$ represents the active and reactive power flow into each node, $\mathrm{Z}$ represents the flow of each of branches, $X$ denotes the voltage amplitude magnitude and phase angle of each node.

Equation (6) written in the form of Taylor series and ignoring the higher order terms gives:

$$
\begin{aligned}
& \Delta X=J_{0}{ }^{-1} \Delta S \\
& \Delta Z=G_{0} \Delta X=G_{0} J_{0}{ }^{-1} \Delta S=T_{0} \Delta S
\end{aligned}
$$

Where, $\mathrm{J}_{0}$ is Jacobi matrix, $\mathrm{G}_{0}$ is the system admittance matrix [11].In the condition of knowing node injection random variable $\Delta S$, the distribution function of branch flow $\Delta Z$ and voltage state variable $\Delta X$ can be obtained by convolution operation.

Firstly, under the premise of the known of k, c, vci, vr, vco and other parameters, each order semi-variable of wind power output can be obtained by formula (5). Then Combined with formula (7), as well as the homogeneous of semi-invariant obtained the each order semi-invariant of node voltage and branch flow. Finally by formula (2), the probability distributions of node voltage and branch flow can be obtained [11].

\section{The Example Analysis of penetration capacity}

This paper chooses IEEE69 node system as example shown in Fig. 1. And each parameter is referred in [12]. 


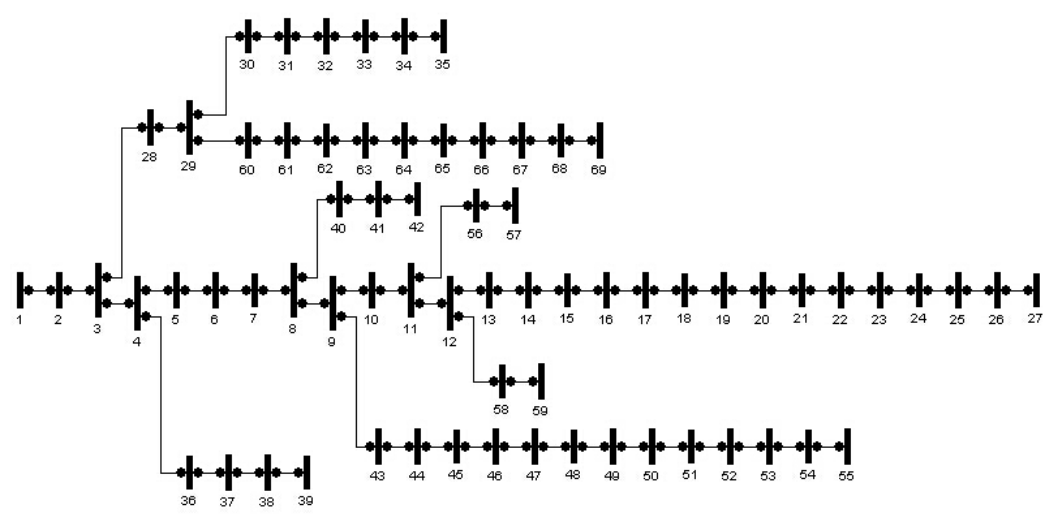

Fig. 1 Schematic of IEEE69 node system

In [13], the calculation model of penetration capacity was established under the premise of wind power keeping constant output. Based on this model, applying probabilistic load flow, and the constraints modified to

$\mathrm{P}($ Uimin $\leq$ Ui $\leq$ Uimax $) \geq \alpha, i \in N$

Where, $\mathrm{N}$ is the set of all the nodes in the distribution network, Uimin and Uimax are the the lower and upper range of static voltage stability range. And $\alpha$ is called confidence level, representing the degree of reliability of static voltage not exceeding which values generally between 0.9 and 1.0.

Setting parameters of wind turbines $\mathrm{k}=2.800383$, $\mathrm{c}=5.141687$, cut-in speed, the rated wind speed, cut-out wind speed respectively vci=3m/s, vr $=14 \mathrm{~m} / \mathrm{s}, \mathrm{vco}=25 \mathrm{~m} / \mathrm{s}$. When $\alpha$ different values, and according to the calculation model with constant power output in [13], the results of penetration capacity are shown in Table 1.

Table 1 penetration capacity results under different circumstances (MW)

\begin{tabular}{|l|l|l|l|l|l|}
\hline Node number & 13 & 16 & 20 & 24 & 27 \\
\hline Constant output & 1.62 & 0.86 & 0.79 & 0.73 & 0.65 \\
\hline$\alpha=0.9$ & 1.32 & 0.68 & 0.62 & 0.58 & 0.52 \\
\hline$\alpha=0.95$ & 1.24 & 0.64 & 0.58 & 0.54 & 0.48 \\
\hline$\alpha=0.98$ & 1.16 & 0.60 & 0.54 & 0.50 & 0.44 \\
\hline$\alpha=1$ & 1.08 & 0.54 & 0.50 & 0.45 & 0.38 \\
\hline
\end{tabular}

It can be drawn from Table 1, when the value of confidence level $\alpha$ becomes larger, each node acceptance of wind power capacity decreases. The higher the confidence level, meaning smaller probability that static voltage exceeding, the stronger the constrains on penetration capacity of wind power, resulting in less wind power capacity. At the same time, the higher confidence level also ensures a higher grid stability, which can demand on the power grid reliability and security to select the appropriate $\alpha$ value. General distribution network power quality requirements are not very strict, allowing static voltage exceeding for a short period of time.Then in order to obtain greater energy efficiency, we can choose a relatively low $\alpha$. For some distribution grid having the more stringent requirements of power quality, it should be the primary goal to ensure the lowest probability of the voltage exceeding.

\section{Conclusion}

In this paper, the characteristics of wind power random output was taken into account in the calculation method of penetration capacity, obtaining the probability distributions of each node voltage by probabilistic load flow. In the premise of ensuring that the probability of a static voltage exceeding less than a certain confidence level $\alpha$, calculating the penetration capacity of wind power. From the results it is clear that the penetration capacity are closely related to the value of confidence level $\alpha$. The value of $\alpha$ closer to 1 , the smaller penetration capacity, the less likely node voltage in the distribution grid exceeding. Therefore, according to the distribution network reliability and 
security requirements, a reasonable choice of confidence level $\alpha$ is necessary.

\section{Acknowledgements}

This work was supported by Science and Technology Foundation of the State Grid Corporation of China for the project in 2014 “ The key technology research and development for dynamic control and operation analysis of wide-area distributed generation”. Hao Bai is the corresponding author.

\section{References}

[1] Li Jianli. Large-scale wind farm on the Influence of Power System Voltage Stability. Qingdao University, 2007.

[2] Zhang Yankai. Research of voltage stability of Grid-connected wind power generation. Lanzhou University of Technology, 2011.

[3] Zou Zhixiang, Zhou Keliang, Yuan Xiaodong. Analysis on Influences of Grid-connected Wind Farm on Voltage Stability of Local Power Network Neighboring to Connection Point. Power System Technology, 2011, 35(11):50-56.

[4] Wang Zhiqun, Zhu Shouzhen, Zhou Shuangxi. Impacts of Distributed Generation on Distribution System Voltage Profile. Automation of Electric Power Systems. 2004, 28(16):56-60.

[5] Zeng Jie,Liu Qisheng,Zhong Jiaojiao.Influence on Static Voltage Stability of System Connected with Wind Power.Power and Energy Engineering Conference(APPEEC),Shanghai.2012 Asia-Pacific.

[6] Anderson Hoke,Rebecca Butler,Joshua Hambrick,Benjamin Kroposki. Steady-State Analysis of Maximum Photovoltaic Penetration Levels on Typical Distribution Feeders.IEEE TRANSACTIONS ON SUSTAINABLE ENERGY, 2013,4(2).

[7] Zhu Xingyang, Liu Wenxia, Zhang Jianhua. Probabilistic Load Flow Method Considering Large-scale Wind Power Integration. Proceedings of the CSEE. 2013, 33(7):77-85

[8] Wang Chengshan, Yu Xuyang. A Method for Computing the Probability Distribution of Fault Critical Clearing Time. Proceedings of the CSEE. 2004, 24(1): 6-10.

[9] Bie Zhaohong, Liu Hui, Li Gan. Voltage Fluctuation of a Power System with Wind Farms Integrated by Probabilistic Load Flow. Journal of Xi'an Jiaotong University. 2008, 42(12):1500-1505.

[10] Zheng Haifeng. Probabilistic Power Flow Containing Distributed Generation In Distribution System. Tianjin University, 2006.

[11] Dong Wei, Yu Jianming, Yang mengmeng. Voltage Security Analysis on the Distribution Network with the Integration of Wind Power by using Stochastic Power Flow. Electric Power. 2012, 45(4):82-86.

[12] Hong Xiaoyu. Service Restoration of Active Distribution Network. Beijing Jiaotong University, 2014.

[13] Xia Chengjun, Cui Hong, Wang Qiang. Penetration Capacity Calculation for Distribution Generation Considering Static Security Constrains. Power System Technology. 2009, 33(16):96-100. 